\title{
A review and application of the failure criteria on a graphite epoxy under three- point bending test
}

\author{
Y. Benbouras*， M. Bellahkim， A. Maziri， E. Mallil， J. Echaabi \\ Équipe de Recherche Appliquée sur les Polymères, Département de Génie Mécanique, ENSEM, Université Hassan II de \\ Casablanca, BP 8118, Oasis, Casablanca, Maroc. \\ *youssef.mecanique@gmail.com
}

\begin{abstract}
The application of failure criteria are difficult partly because they are too various and must be validated by biaxial tests, which are expensive to be achieved. In this work, an analytical modeling based on a software program has been elaborated for application of the most general failure criteria and prediction of successive failure. Finally, the results obtained by this analytical modeling show a good correlation with those carried out by experimental study.
\end{abstract}

Keywords: Three-point bending test, Failure criteria, successive failure.

\section{Introduction}

The orthotropic material strengths parameters are typically established by test prediction in principal material direction. Generally, beams and plates are easily subjected to in-plane multiaxial stress states during service. This stress depends on the stacking sequence and the physical properties of resin and fiber mixture. It should be noted that, the in-plane shear stress may play a significant role in the failure [4]. Consequently, a number of failure criteria have been developed in order to predict failure loads under arbitrary stress states. These failure criteria can be grouped into two main groups.

- Failure criteria neglecting interactions between different stress components.

- Failure criteria considering interactions between different stress components.

In our case, both types were used and we also integrated into our study the failure criteria recently published.

Three points bending test is a useful and simple experiment often used to determinate the mechanical properties and failure criteria for composite laminates. Many authors were interested in this test [8-10] . In this work, we used and improved the experimental results, developed by Echaabi [5], by prediction of successive failure.

\section{Brief overview of failure criteria 2-1 Maximum stress criteria}

Failure criteria, neglecting interactions between different stress components, are the simplest and they usually propose one inequality for each one of the three in-plane stresses (or strain) components. Maximum stress criteria belong to this category [4]. If the fibers are taken parallel to axis 1 and the main referential of the material is considered. Thus, the conditions for failure can be stated as

$$
\begin{aligned}
& \sigma_{11}>X^{T} \text { or } \sigma_{11}<-X^{C} \\
& \sigma_{22}>Y^{T} \text { or } \sigma_{22}<-Y^{C} \\
& \tau_{12}>S^{L}
\end{aligned}
$$

Figure 1 gives an example of failure envelope of maximum stress criterion. The envelope related to this criterion is obtained through neglecting the Shear stress interaction and the linear transformation by Hooke's matrix. Practically, this mathematical relation could not be rigorously satisfied [4].

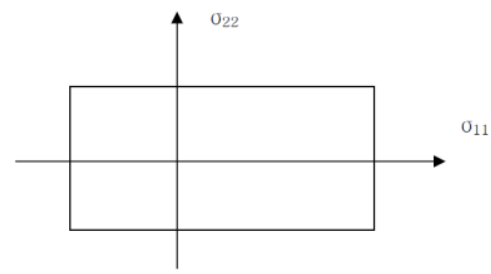

Figure 1. Failure envelope of maximum stress criterion

\section{2-2 Tsai-Hill criterion}

The Tsai-Hill is an interactive criterion that takes into account the effect of the in-plane shear stress[4].This failure criterion was formulated by referring to distortional energy, thereby, the condition for failure is given by the following inequality:

$\left(\frac{\sigma_{11}}{X}\right)^{2}-\left(\frac{\sigma_{11} \sigma_{22}}{X^{2}}\right)+\left(\frac{\sigma_{22}}{Y}\right)^{2}+\left(\frac{\tau_{12}}{S^{L}}\right)^{2}>1$

Where the failure parameters $\mathrm{X}$ and $\mathrm{Y}$ depend on the considered quadrant of the coordinate plane, being

$X=X^{T}$ or $X=X^{c}$ if $\sigma_{11}>0$ or $\sigma_{11}<0$, respectively and $Y=Y^{T}$ or $Y=Y^{c}$ if $\sigma_{22}>0$ or $\sigma_{22}<0$.

Figure 2 shows an example of corresponding failure envelopes with varying $\tau_{12}$. The in-plane shear stress contributes to the decrease of the semimajor and semiminor axes of the four ellipses composing the plots 1 .

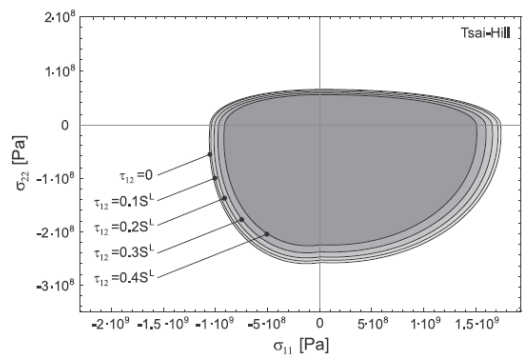

Figure 2. Failure envelopes of Tsai-Hill criterion by varying $\tau_{12}$ 


\section{2-3 Tsai-Wu criterion}

Tsai-Wu criterion is an interactive approach considering in-plane shear stress effects. This failure criterion was formulated in order to fit experimental results. In addition, it was not derived from a physical basis. [4]:

$$
\begin{aligned}
& \left(\frac{1}{X^{T}}-\frac{1}{X^{C}}\right) \sigma_{11}+\left(\frac{1}{Y^{T}}-\frac{1}{Y^{C}}\right) \sigma_{22}+\frac{\sigma_{11}^{2}}{X^{T} X^{C}} \\
& +\frac{\sigma_{22}^{2}}{Y^{T} Y^{C}}+\frac{\tau_{22}^{2}}{S^{L} S^{L}}-\frac{\sigma_{11} \sigma_{22}}{\sqrt{X^{T} X^{C} Y^{T} Y^{C}}}>1
\end{aligned}
$$

The same result such as the of Tsai-Hill criterion, the semimajor and semiminor axes of the corresponding ellipse are reduced by the in-plane shear stress effect

\section{2-4 Hashin criterion}

Interactive and non-interactive conditions were proposed by Hashin criterion, in order to differentiate between matrix or fiber failure caused by tension or compression. Sun et al $[1,2]$ was proposed a further version according to some empirical modifications. Consequently, the Hashin criterion is interactive and the conditions for failure being given by the following inequalities.

Fiber failure for tension $\left(\sigma_{11} \geq 0\right)$

$\left(\frac{\sigma_{11}}{X^{T}}\right)^{2}+\left(\frac{\tau_{12}}{S^{L}}\right)^{2} \geq 1$

Matrix failure for tension $\left(\sigma_{22}<0\right)$

$$
\left(\frac{\sigma_{22}}{2 S^{L}}\right)^{2}+\left(\left(\frac{Y_{C}}{2 S^{L}}\right)^{2}-1\right) \frac{\sigma_{22}}{Y^{C}}+\left(\frac{\tau_{22}}{S^{L}}\right)^{2} \geq 1
$$

Matrix failure for compression $\left(\sigma_{22} \geq 0\right)$

$$
\left(\frac{\sigma_{22}}{Y^{T}}\right)^{2}+\left(\frac{\tau_{12}}{S^{L}}\right)^{2} \geq 1
$$

Fiber failure for compression $\left(\sigma_{11}<0\right)$

$$
-\frac{\sigma_{11}}{X^{C}} \geq 1
$$

\section{2-5 LaRC03 criterion}

The LaRC03 failure criterion suggests a combination of five interactive conditions in order to indicate a crack initiation in the matrix or a failure due to fiber compression. This failure criterion was developed after the WWFE at Langley Research Center [3]. Only for tensional fiber failure a noninteractive inequality is suggested. Thereby, a summary of the $\mathrm{LaRC} 03$ criterion is proposed in the following.

Matrix cracking for tension $\left(\sigma_{22} \geq 0\right)$ :

$$
(1-\mathrm{g})\left(\frac{\sigma_{22}}{Y_{i s}^{T}}\right)+g\left(\frac{\sigma_{22}}{Y_{i s}^{T}}\right)^{2}+\left(\frac{\tau_{12}}{S_{i s}^{L}}\right)^{2} \geq 1
$$

With $g=1,12^{2} \frac{A_{22}^{0}}{A_{44}^{0}}\left(\frac{Y^{T}}{S^{L}}\right)^{2}, \mathrm{~A}_{22}^{0}=2\left(\frac{1}{E_{2}}-\frac{v_{21}^{2}}{E_{1}}\right)$ and $\mathrm{A}_{44}^{0}=\frac{1}{G_{12}}$. In the case of thick plates, the following formulae can be used:

$$
\mathrm{Y}_{i s}^{T}=1.12 \sqrt{2} Y^{T} \text { and } S_{i s}^{L}=\sqrt{2} S^{L} \text {. }
$$

Matrix cracking for compression $\left(\sigma_{11}<0\right)$ :

$$
\left(\frac{\tau_{e f f}^{T}}{S^{L}}\right)^{2}+\left(\frac{\tau_{e f f}^{T}}{S_{i s}^{L}}\right)^{2} \geq 1
$$

Where the effective shear stresses $\tau_{\text {eff }}^{T}$

$$
\begin{aligned}
& \tau_{\text {eff }}^{T}=\left|-\sigma_{22} \cos (\alpha)\left[\sin (\alpha)-\mathrm{n}^{T} \cos (\alpha)\right]\right| \\
& \text { and } \mathrm{n}^{T}=\frac{-1}{\tan \left(2 \alpha_{0}\right)}
\end{aligned}
$$

Failure for fiber tension $\left(\sigma_{11} \geq 0\right)$ :

$$
\frac{\varepsilon_{11}}{\varepsilon_{11}^{T}} \geq 1
$$

Failure for fiber compression $\left(\sigma_{22}<0\right)$

$$
\left\langle\frac{\left\lfloor\tau_{12}^{m}\right\rfloor+n^{T} \sigma_{22}^{m}}{S_{i s}^{L}}\right\rangle \geq 1
$$

Usually, $\alpha_{0}=53^{\circ}$ from test data 1-16 (see Figure.11). As suggested in work 20-21 within a loop over the range of possible fracture angles between $0^{\circ}$ and $\alpha_{0}=53^{\circ}$.

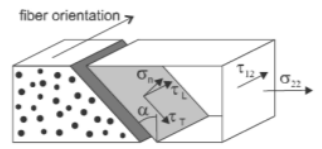

Figure 3. Angle of the fracture plane of a unidirectional lamina subjected to transverse compression and in-plane shear.

The Hashin criterion indicates that resizing occurs with respect to a point located along the envelope's edge pertaining to the fiber compression. Moreover, $\mathrm{LaRC0} 3$ criterion shows that the center of resizing is a point along the envelope's edge related to fiber failure due to traction (Figure 4) [4].

In the next section, we tested these failure criteria in the case of three-point bending test.
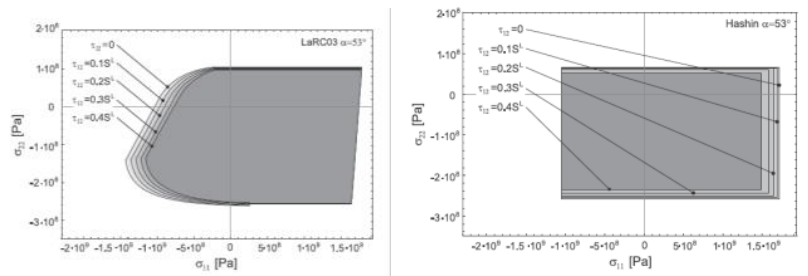

Figure 4. Failure envelopes of Hashin and $\mathrm{LaRC} 03$ criterion by varying $\tau_{12}$

\section{Simulation of the failure}

To test the failure criteria cited and our failure theory algorithm, a graphite-epoxy unidirectional fiber-reinforced with staking sequence $\left[[+45 /-45 / 90 / 0]_{3}\right]_{\mathrm{s}}$ is considered in the following. Its mechanical characteristics are [5]: $\mathrm{E} 11=116 \mathrm{GPa}, \mathrm{E} 22=\mathrm{E} 33=6.9 \mathrm{GPa}, \mathrm{G} 12=5.6 \mathrm{GPa}$, $\mathrm{G} 13=3.4 \mathrm{GPa}, \mathrm{G} 23=2.5 \mathrm{GPa}, v 12=v 13=v 23=0.3$.

After comparison between the experimental macroscopic curves of the test specimens with matrix cracking observed, we noticed that the final failure occurs only when a fiber oriented $0^{\circ}$ is damaged. For that, our methodology used for a progressive failure analysis is illustrated in Figure 5. Failure theory algorithm was developed by VBA/Excel application. The deflection causing the damage allows the calculation of the force applied using the nonlinear approach of Venetis [6]. 
Thus, the analytical results are combined with the criteria of the maximum stress, Hashin, LaRC03 and Tsai Hill enables us to reduce the error margin to $6 \%$ compared to the experimental results. On the other hand, Tsai-Wu criterion overestimates failure. This is shown in Table 1.

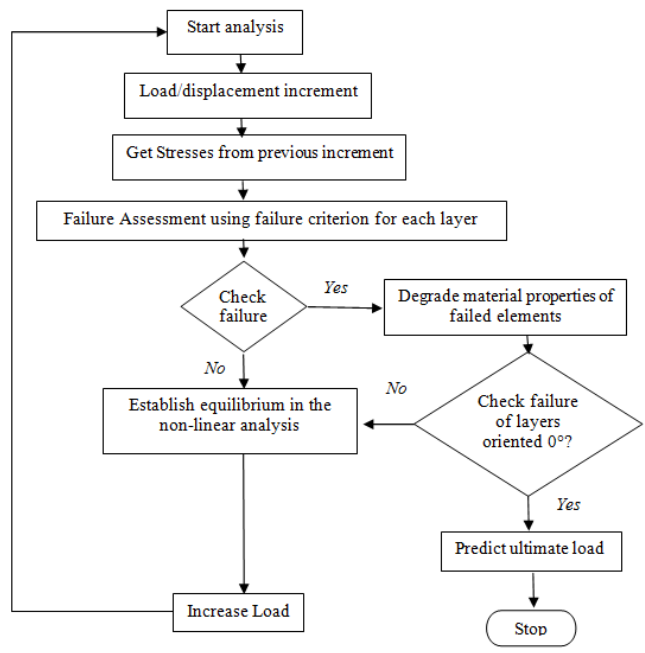

Figure 5. Progressive failure algorithm

To interpret the results shown in table 1 . We have thoroughly analyzed the angle of the fracture of the specimens tested.

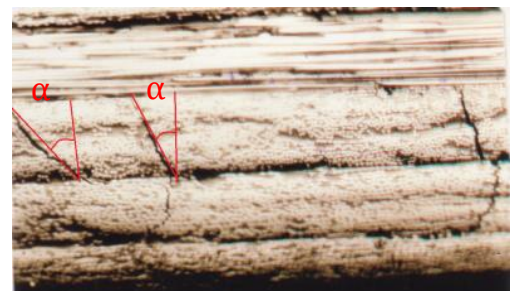

Figure 6. Angle of the fracture related to specimen with staking sequence [[+45/-45/90/0]3]s - Layer 2 and 3. [5]

We can notice that, in the case of three point bending test, the angle of the fracture related to specimens is on the order of $53^{\circ}$. This shows that the specimen is subject a pure transverse compression/tensile. In addition, a study was conducted by WWFE [7] shows that the criterion converges towards the same results in the case of $\tau_{12}=0$ (Figure 7).This explains the strong correlation observed between the different failure criteria tested (Table 1).

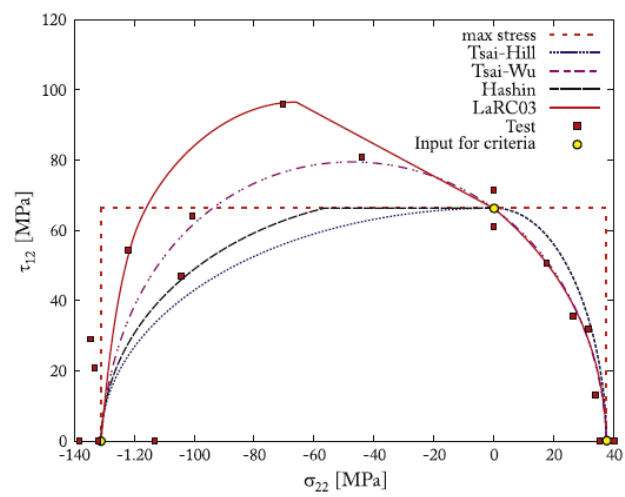

Figure 7. Comparison among failure envelopes and WWFE test data for unidirectional composite E-Glass/LY556.

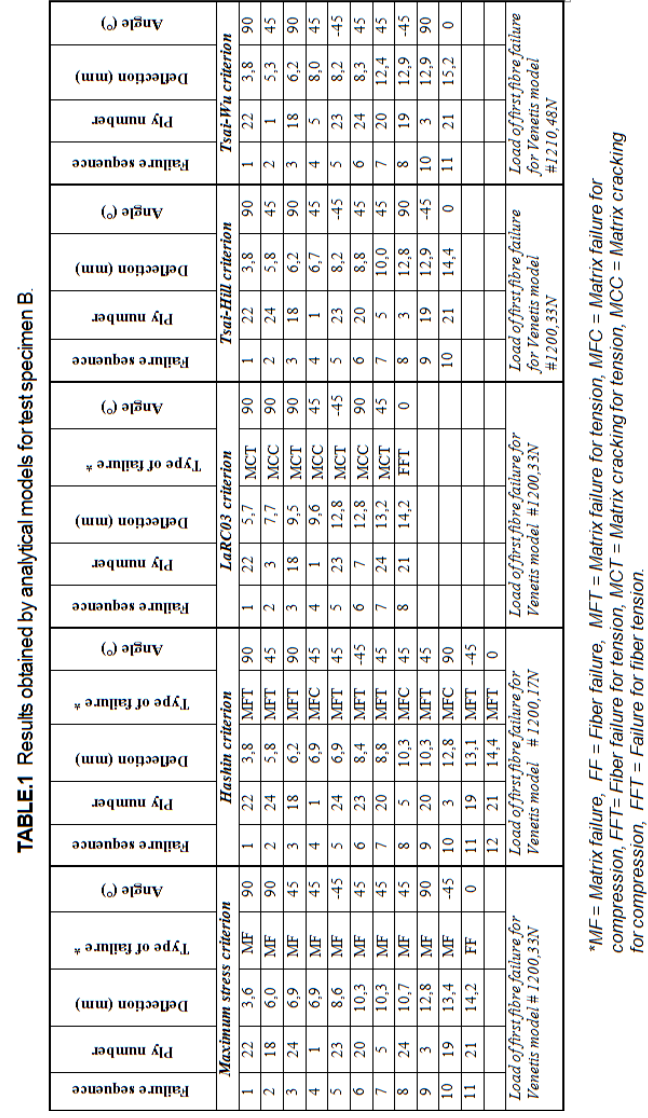

\section{Conclusion}

The prediction of the succession of the failure and the first macroscopic failure was well estimated by maximum strain, Hashin, LaRC03 and Tsai Hill criteria. But, Tsai-Wu criterion overestimates failure. In addition, all the failure criterion converges towards the same results in the case of $\tau_{12}=0$.

\section{References}

[1] Z. Hashin and A. Rotem, "A Fatigue Failure Criterion for Fiber Reinforced Materials," J. Compos. Mater., vol. 7, no. 4, pp. 448464, Oct. 1973

[2] Z. Hashin, "Failure Criteria for Unidirectional Fiber Composites," J. Appl. Mech., vol. 47, no. 2, p. 329, Jun. 1980

[3] C. Davila, N. Jaunky, and S. Goswami, "Failure Criteria for FRP Laminates in Plane Stress," in 44th AIAA/ASME/ASCE/AHS/ASC Structures, Structural Dynamics, and Materials Conference, 2003.

[4] P. Nali, E. Carrera "A numerical assessment on two-dimensional failure criteria for composite". Composites: Part B.vol.43. P 280 289. (2012).

[5] J. Echaabi, F. Trochu, X. T. Pham, and M. Ouellet, "Theoretical and Experimental Investigation of Failure and Damage Progression of Graphite-Epoxy Composites in Flexural Bending Test," J. Reinf. Plast. Compos., vol. 15, no. 7, pp. 740-755, Jul. 1996.

[6] Y. Benbouras, A. Maziri, E. Mallil, and J. Echaabi, "A NONLINEAR ANALYTICAL MODEL FOR SYMMETRIC LAMINATED," International Journal of Technology, vol., 8, no. 6, pp. 437-447, 2017.

[7] Davila CG, Camanho PP, (2003). Failure criteria for frp laminates in plane stress.Tech. Rep. NASA/TM,2003-212663.

[8] GustavoVargasFaustinoMujika, "Determination of in-plane shear properties by three-point flexure test of $\pm 45^{\circ}$ anti-symmetric laminates," Polym. Test., vol. 30, no. 2, pp. 204-215, Apr. 2011.

[9] XiwenJiaaZihuiXiabBohongGua, "Numerical analyses of 3D orthogonal woven composite under three-point bending from multiscale microstructure approach," Comput. Mater. Sci., vol. 79, pp. 468-477, Nov. 2013

[10] M.C.Serna MorenoA.Romero GutiérrezJ.L.Martínez Vicente, "Different response under tension and compression of unidirectional carbon fibre laminates in a three-point bending test," Compos. Struct., vol. 136, pp. 706-711, Feb. 2016. 